\title{
Least Square and Min-Max Design of MTI Filters With Nonuniform Interpulse Periods
}

\author{
Mehmet İspir \\ İLTAREN-BILGEM \\ TÜBİTAK \\ Ankara, Turkey \\ mehmet.ispir@tubitak.gov.tr
}

\author{
Çağatay Candan \\ Middle East Technical University (METU) \\ Department of Electrical and Electronics Engineering \\ Ankara, Turkey \\ ccandan@metu.edu.tr
}

\begin{abstract}
The design of moving target indicator (MTI) filters with nonuniform interpulse periods is studied through the least square and min-max filter design methodologies. A trade-off between the contradictory objectives of maximum possible clutter suppression (maximum stopband attenuation) and minimum desired signal attenuation (minimum passband ripple) is established by the introduction of a weight factor into the design. The weight factor enables the adaptation of MTI filters to different operational scenarios such as system operation under low, medium or high clutter power.
\end{abstract}

\section{INTRODUCTION}

Staggering the interpulse periods is a widely used solution to improve the blind speed problem in moving target indicator (MTI) radars. It is possible to increase the first blind speed on the order of ten folds (or more) with the use of staggered pulse repetition intervals (PRI). It should be noted that the improvement in blind speed comes at almost no additional computational cost apart from losing the utilization of Fast Fourier Transform (FFT). An important disadvantage of nonuniform sampling is the passband ripples which are much larger in comparison to uniform PRI systems. Hence, the fluctuations of signal power at the MTI filter output may degrade the detection performance for some Doppler frequencies. Our goal in this paper is to apply classical filter design techniques, such as the least square and min-max filter design methods, to the MTI filter design.

Staggered MTI systems have been studied in the literature since 1970's, [1]-[10]. The application of staggered MTI is especially attractive for systems operating under low PRF (pulse repetition frequency) regimes such as weather surveillance radars, [5].

The design of staggered systems comprises on the optimization of two sets of parameters: the interpulse time durations and filter coefficients. The unambiguous range and velocity specifications impose constraints on the interpulse periods. The amount of desired clutter suppression (improvement factor specification), along with the interpulse periods, affects the choice of filter coefficients. In this paper, we focus on the filter design problem and assume that the interpulse periods are provided to us. Our goal is to present a flexible solution to the MTI filter design for a given set of interpulse periods. It should be noted that the results of this paper can be used in the selection of the best interpulse periods from a set of suitable candidates satisfying the unambiguous range-velocity constraints.

The goal of MTI filter design is to provide maximum amount of clutter suppression concurrently with the least amount desired signal suppression (flat passband). Figure 1 illustrates the desired response.

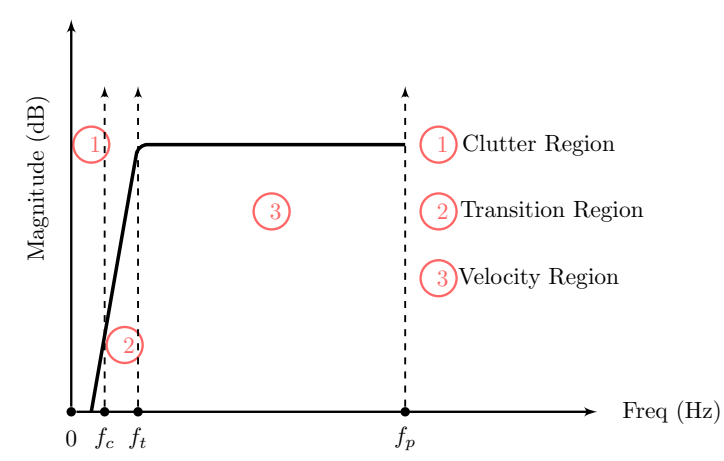

Fig. 1. Desired Frequency Response for MTI Filters

Majority of the MTI filter designs in the literature are specifically developed for surveillance and weather radar systems, [1], [2], [4], [5]. Some related work on nonuniformly sampled signals are also presented in the literature, [6], [7]. Among these methods, some prominent ones are as follows: The method developed by Prinsen [1] adjusts the filter weights to provide maximally flat stopband characteristics at the zero frequency. The approach of Prinsen relies on the Taylor series expansion of the frequency response at the zero frequency and can be considered as the dual of single line canceller systems with the transfer function $H(z)=1-z^{-1}$, for nonuniform PRI systems. At a later work, Prinsen has suggested the optimization of the stagger periods with the filter weights, [4]. In [2], Hsiao has suggested the optimization of the filter weights with the objective of the flat passband along with the constraint of maximum stopband attenuation. The work of Hsiao does not enable a trade-off between the desired responses in passband and stopband. Jacomini uses the constraints of Hsiao; but suggests a change in the cost function to assist the computation of the filter discussed in [3]. 
In the present work, we study two widely adopted FIR filter design techniques, namely the least square and min-max filter design, on the optimization of nonuniform MTI filters and compare the designs with the available ones in the literature.

\section{NONUNIFORM MTI FiLTER DESIGN}

The structure of the discrete FIR type staggered MTI filter is given in Figure 2.

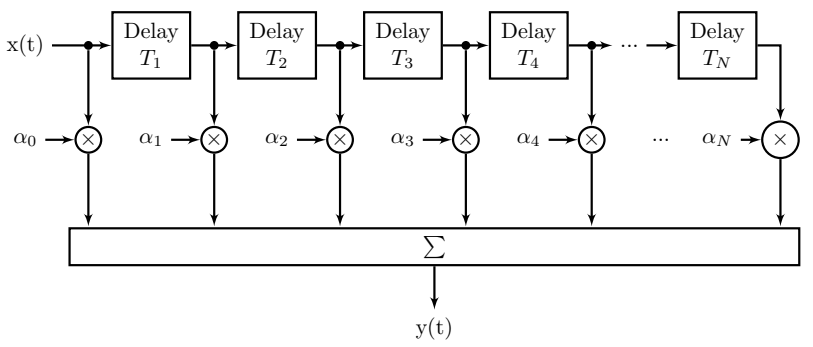

Fig. 2. FIR Filtering with Nonuniform Delays

As shown in Figure 2, the output signal $y(t)$ is formed by a linear combination of the nonuniformly sampled input signal. The impulse response of this system can be expressed as follows:

$$
h(t)=\sum_{n=0}^{N} \alpha_{n} \delta\left(t-t_{n}\right)
$$

Here $\alpha_{n}$ is the n'th filter coefficient and $t_{n}$ is the n'th sampling time which is given as follows:

$$
t_{n}= \begin{cases}\sum_{i=0}^{n-1} T_{i}, & n \geq 1 \\ 0, & n=0\end{cases}
$$

It should be noted that $T_{i}$ 's correspond to the interpulse periods for the examined radar signal processing application. The frequency response of the filter given in (1) can be written as follows:

$$
H(f)=\sum_{n=0}^{N} \alpha_{n} e^{-j 2 \pi f t_{n}}
$$

Here $f$ denotes the frequency in Hertz.

Figure 1 shows different frequency bands of interest for the MTI filter design [11]. The Clutter Region denotes the band where the clutter signal power is essentially located. The upper limit of this region defines the cutoff frequency of filter $\left(f_{c}\right)$. The Transition Region is related with the steepness of the filter response between stopband and passband. The transition region is also denoted as "don't care" region in the filtering literature. The Velocity Region is the band of Doppler frequencies for the targets of interest. This band is also called as the filter passband.

The objectives of the MTI filter design can be listed as follows:

- Minimizing the passband ripple in velocity region

- Maximizing the stopband attenuation in clutter region

It should be clear that both objectives can not be achieved simultaneously and a practical solution has to operate at a trade-off between these objectives. Our goal in this work is to apply general filter design frameworks to the MTI filter design and present a flexible method capable of trading objectives with each other.

Before the examination of filter design methods, we would like to present some criterion that would be useful in the comparison of designs.

Stopband Attenuation: This is the value of filter magnitude response at the cutoff frequency $f_{c}$. Since the frequency values smaller than $f_{c}\left(0 \leq f \leq f_{c}\right)$ are typically attenuated more than the value at the cut-off frequency, this value can be considered to represent the worst case signal attenuation in the stopband.

Maximum Deviation: This criteria indicates the maximum magnitude deviation from the ideal response in the velocity region.

Mean Passband Error: This criteria is a measure for the flatness of the filter response in the velocity region:

$$
\epsilon=\int_{f_{t}}^{f_{p}}\left|H_{d}(f)-H(f)\right|^{2} d f
$$

Here $H_{d}(f)$ and $H(f)$ are the frequency responses of the ideal and designed filters respectively. The lower and upper limits of the integral in (4) are the lower and upper limits of velocity region. The presented criterion are illustrated in the Figure 3.

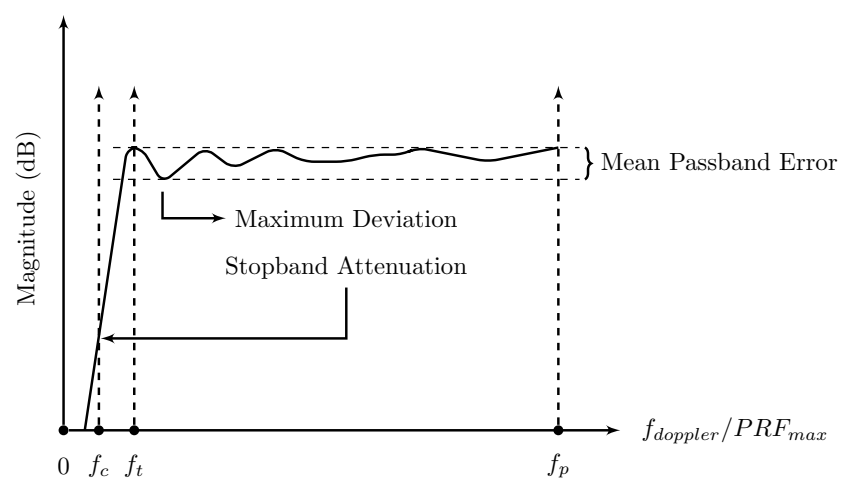

Fig. 3. Criterion for Filter Comparison

The presented criterion are used to compare the magnitude response of filters. In order to examine the clutter attenuation performance, MTI improvement factor can be utilized. MTI improvement factor is defined as "the signal-to-clutter power ratio at the output of the MTI filter to the signal-to-clutter power ratio at the input, averaged uniformly over all target velocities of interest." [12] and can be expressed as

$$
\text { Improvement Factor }=I F=\frac{(S C R)_{\text {out }}}{(S C R)_{\text {in }}}
$$

where $S C R_{\text {out }}$ and $S C R_{\text {in }}$ represent average signal-to-clutter ratio at the output and input of the MTI filter respectively. Improvement factor can be considered as the average SCR improvement of the MTI filter. 


\section{A. Least Square (LS) Design}

The standard cost equation for the least square filter is given as follows:

$$
J_{\text {cost }}=\int_{0}^{f_{p}}\left|H_{d}(f)-H_{l s}(f)\right|^{2} d f
$$

Here $H_{d}(f)$ and $H_{l s}(f)$ denotes the frequency responses of the desired and the approximation to the desired filter in the least square sense, that is

$$
H_{d}(f)= \begin{cases}0, & f<f_{c} \\ 1, & f \geq f_{c}\end{cases}
$$

and

$$
H_{l s}(f)=\sum_{k=0}^{N-1} \alpha_{i} e^{-j 2 \pi f t_{k}} .
$$

Here $f_{c}$ is the cutoff frequency of the desired filter, $\alpha_{i}$ 's are the filter coefficients and $t_{i}$ 's are the sampling times.

To suppress the DC clutter, $\sum_{i=0}^{N-1} \alpha_{i}=0$ constraint can be used turning the least square design into a constraint least square design. By including the constraint equation into the design via Lagrangian Multiplier $\lambda$, the cost equation can be rewritten as follows:

$J_{\text {cost }}(\alpha, \lambda)=\int_{0}^{f_{p}}\left|H_{d}(f)-\sum_{i=0}^{N-1} \alpha_{i} e^{-j 2 \pi f t_{i}}\right|^{2} d f+\lambda\left(\sum_{i=0}^{N-1} \alpha_{i}\right)$

By taking the partial derivatives of the cost function with respect to filter coefficients, we can get the following equation:

$$
\frac{\partial J_{\text {cost }}}{\partial \alpha_{i}^{*}}=\int_{0}^{f_{p}}\left(H_{d}(f)-\sum_{n=0}^{N-1} \alpha_{n} e^{-j \pi f t_{n}}\right) e^{j 2 \pi f t_{i}} d f+N \lambda
$$

By equating, the partial derivatives given in (10) to zero for $i=\{0,1, \ldots, N\}$, the following linear equation system can be written:

$$
\mathbf{A} \boldsymbol{\alpha}=\mathbf{H}_{\mathbf{d}}+\lambda \mathbf{1}
$$

Here 1 is the $N \times 1$ column vector with entries of $1, \mathbf{H}_{\mathbf{d}}$ is a $N \times 1$ column vector with the k'th entry $\mathbf{H}_{\mathbf{d}}(k)=$ $\int_{f_{c}}^{f_{p}} e^{j 2 \pi f t_{k}} d f$ and $\mathbf{A}$ is a $N \times N$ matrix with the i'th row and $\mathrm{j}$ 'th column entry $\mathbf{A}(i, j)=\int_{0}^{f_{p}} e^{-j 2 \pi f\left(t_{j}-t_{i}\right)} d f$. Finally, the vector $\boldsymbol{\alpha}$ in (11) is the vector unknowns, that is the MTI filter coefficients.

In order to establish a trade-off between the objectives of clutter attenuation and passband ripple; we introduce a weight $\mathcal{W}$ to control the contribution of stopband error to the cost function. The weight $\mathcal{W}$ changes the cost given in (9) as follows:

$$
\begin{aligned}
J_{\text {cost }}^{\mathcal{W}} & =\mathcal{W} \int_{0}^{f_{c}}\left|H_{d}(f)-H_{l s}(f)\right|^{2} d f \\
& +\int_{f_{t}}^{f_{p}}\left|H_{d}(f)-H_{l s}(f)\right|^{2} d f+\lambda\left(\sum_{i=0}^{N-1} \alpha_{i}\right)
\end{aligned}
$$

Optimization with the weighted cost function results in the following equation for the calculation of the least square design filter coefficients:

$$
\left(\mathcal{W} \times \mathbf{A}_{\text {stop }}+\mathbf{A}_{\text {pass }}\right) \boldsymbol{\alpha}=\mathbf{H}_{\mathbf{d}}+\lambda \mathbf{1}
$$

In the last equation, we have $\mathbf{A}_{\text {stop }}(i, j)=$ $\int_{0}^{f_{c}} e^{-j 2 \pi f\left(t_{j}-t_{i}\right)} d f$ and $\mathbf{A}_{\text {pass }}(i, j)=\int_{f_{t}}^{f_{p}} e^{-j 2 \pi f\left(t_{j}-t_{i}\right)} d f$. It should be clear that by increasing $\mathcal{W}$, the contribution of the stopband error to the cost function is increased and therefore, the optimized filter provides more clutter suppression for higher $\mathcal{W}$ values.

The filter coefficients of the least square design $(\boldsymbol{\alpha})$ can be calculated by rewriting (13) as follows:

$$
\boldsymbol{\alpha}=\left(\mathcal{W} \times \mathbf{A}_{\text {stop }}+\mathbf{A}_{\text {pass }}\right)^{-1}\left(\mathbf{H}_{\mathbf{d}}+\lambda \mathbf{1}\right)
$$

In order to find the least square MTI filter coefficients, Lagrangian Multiplier $\lambda$ must be written in terms of other variables. Since $\mathbf{1}^{\mathbf{T}} \boldsymbol{\alpha}=0$, Lagrangian Multiplier $\lambda$ can be found as follows:

$$
\begin{aligned}
\mathbf{1}^{\mathbf{T}} \boldsymbol{\alpha}= & \mathbf{1}^{\mathbf{T}}\left(\mathcal{W} \times \mathbf{A}_{\text {stop }}+\mathbf{A}_{\text {pass }}\right)^{-1} \mathbf{H}_{\mathbf{d}} \\
& +\mathbf{1}^{\mathbf{T}}\left(\mathcal{W} \times \mathbf{A}_{\text {stop }}+\mathbf{A}_{\text {pass }}\right)^{-1} \lambda \mathbf{1}=0 \\
\lambda & =\frac{\mathbf{1}^{\mathbf{T}}\left(\mathcal{W} \times \mathbf{A}_{\text {stop }}+\mathbf{A}_{\text {pass }}\right)^{-1} \mathbf{H}_{\mathbf{d}}}{\mathbf{1}^{\mathbf{T}}\left(\mathcal{W} \times \mathbf{A}_{\text {stop }}+\mathbf{A}_{\text {pass }}\right)^{-1} \mathbf{1}}
\end{aligned}
$$

\section{B. Min-Max Design}

In this section, we examine the min-max filter design method for MTI filter design. The min-max filter design aims to select the filter coefficients such that the maximum deviation from the desired response is minimized. The optimization problem can be stated as follows:

$$
\begin{array}{ll}
\operatorname{minimize} & \delta \\
\text { subject to } & \left|H_{m m}\left(f, \alpha_{i}\right)\right| \leq \delta, \quad f \in\left[0, f_{c}\right], \\
& |1-| H_{m m}\left(f, \alpha_{i}\right) \mid \leq \mathcal{W} \delta, \quad f \in\left[f_{t}, f_{p}\right], \\
& \left(\alpha_{i} \in \mathbf{R}, \quad i \in \mathbf{N}_{\mathbf{0}}\right) \\
& \sum_{n=0}^{N-1} \alpha_{n}=0, \quad \alpha_{i} \in \mathbf{R}
\end{array}
$$

Here $H_{m m}(f)$ is the frequency response of min-max filter and the variable $\delta$ shows the maximum deviation from the desired characteristics (for $\mathcal{W}=1$ ). The goal in this design is to minimize the maximum deviation from the desired highpass characteristic. The first and second constraints enforce the magnitude deviation be smaller than $\delta$ (for $\mathcal{W}=1$ ) in the designated bands. The third constraint guarantees that the min-max design has a null at DC frequency.

Different from the LS design, there is no closed form mathematical relation from which the optimal min-max filter coefficients can be retrieved. The optimization has to be done numerically. The numerical implementation of the optimization problem requires the discretization of frequency band into a dense set of frequency points. Therefore, the constraints given in (17) are not evaluated for a continuum of points; 
but for a finitely many number of points. Discretization of the frequency band is realized by uniformly dividing the frequency band with respect to specified frequency resolution and the constraints are evaluated for these frequency values. More details on numerical optimization and additional simulation results can be found in [13].

Similar to the LS design, a weight factor $\mathcal{W}$ is introduced to establish a trade-off between clutter attenuation and passband ripple objectives. It should also be noted that the min-max problem examined here is focused on minimizing the deviation of the magnitude response from the desired response. In the literature, the problem of filter design to match a desired magnitude response is studied for uniformly sampled signals in [14], but not for the nonuniformly sampled signals.

\section{NUMERICAL COMPARISONS}

In this section, we present the results of numerical comparisons. Here the designed filters are compared between each other and also with other filters in the literature. We take the optimized filters of Hsiao and Jacomini as reference designs from the literature, [2], [3]. In addition to these filters, we also compare the designs with the Prinsen filter given in [1] and with the standard MTI filter, i.e. the binomial filter having the transform function of $\left(1-z^{-1}\right)^{N}$.

It should be noted that the staggering periods given in Hsiao and Jacomini papers are different from each other. We individually compare the designed filters with the optimized filters of Hsiao and Jacomini. As an example, the staggering ratios of $[279,204,150,175,230]$ appearing in the title of Figure 5 are taken from [3] and represent the interpulse periods in miliseconds. The corresponding sampling times $t_{k}$ for the scenario of Jacomini becomes $[0.0,0.279,0.483,0.633,0.808,1.047]$ seconds.

Tables I and II present the coefficients of MTI filters for different designs. (The weight parameter for the proposed filters are also given in the tables.) The frequency responses of the filters are given in Figures 4 and 5. The lower panel of the frequency response figures show the zoomed version of the stopband.

Tables III and IV present the numerical values for the criterion defined earlier. It can be noted from these tables that the min-max filter present some advantage in comparison to other filters including the optimized filters of Hsiao and Jacomini in all cases.

TABLE I

MTI FILTERs For INTERPULSE PERIODS GIVEN IN [2]

\begin{tabular}{|l|c|c|}
\hline & $\mathcal{W}$ & Normalized Filter Coeff. (SR: 111110121411 ) \\
\hline Ls & $10^{4}$ & $0.1003,-0.3418,0.6104,-0.6036,0.3382,-0.1424,0.0390$ \\
\hline Min-Max & $10^{4}$ & $0.0793,-0.2969,0.5753,-0.6174,0.3872,-0.1967,0.0693$ \\
\hline Hsiao & - & $0.0809,-0.3011,0.5846,-0.6173,0.3796,-0.1795,0.0693$ \\
\hline Prinsen & - & $0.0381,-0.2270,0.5760,-0.6689,0.3868,-0.1321,0.0271$ \\
\hline Binom & - & $0.0329,-0.1974,0.4935,-0.6580,0.4935,-0.1974,0.0329$ \\
\hline
\end{tabular}
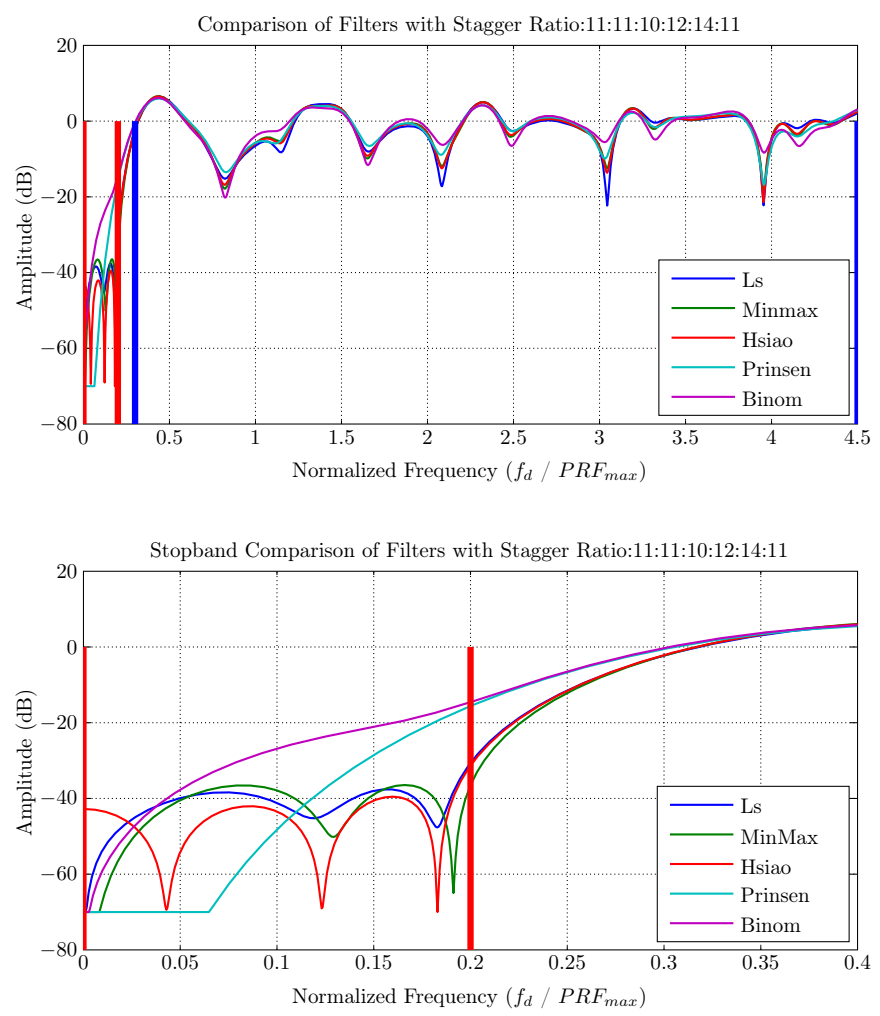

Fig. 4. MTI Filter Frequency Response For Interpulse Periods of [2].
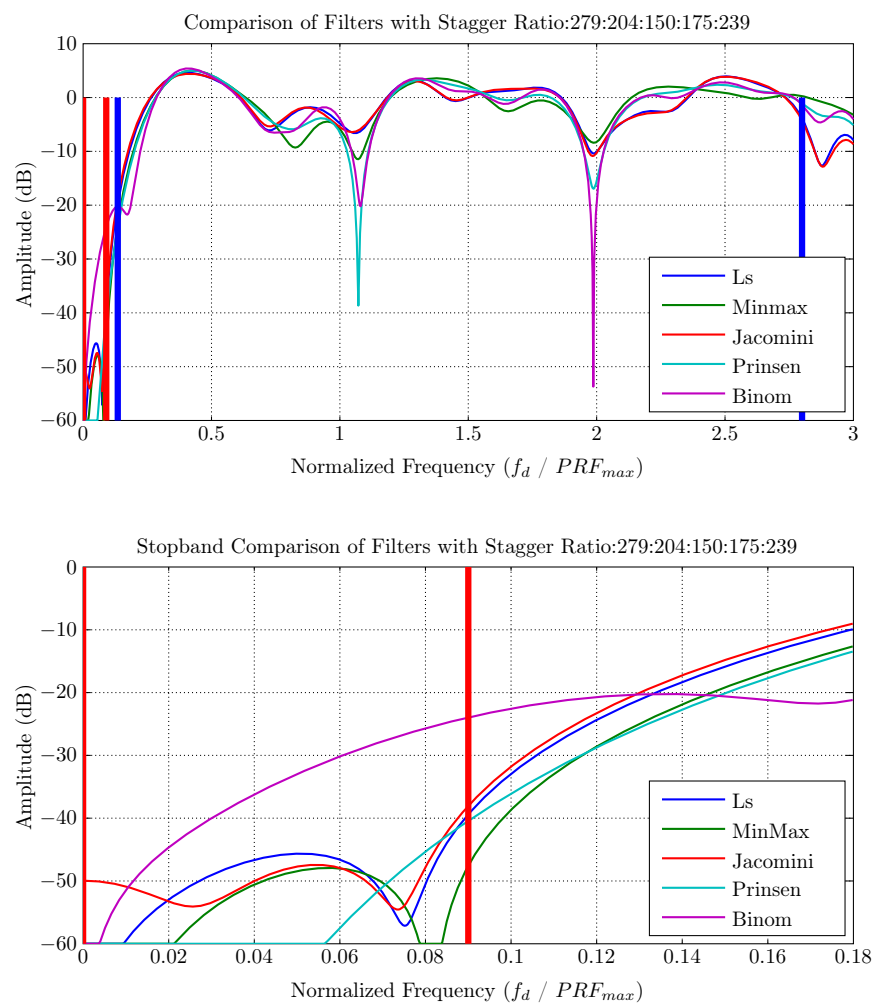

Fig. 5. MTI Filter Frequency Response For Interpulse Periods of [3]. 
TABLE II

MTI Filters For INTERPUlse PERIOds Given IN [3]

\begin{tabular}{|l|c|c|}
\hline & $\mathcal{W}$ & Normalized Filter Coeff. (SR:279 204 150 175 209) \\
\hline LS & $10^{4}$ & $0.0760,-0.3731,0.7290,-0.5562,0.1188,0.0054$ \\
\hline Min-Max & $10^{5}$ & $0.0018,-0.1200,0.5317,-0.7355,0.3956,-0.0737$ \\
\hline Jacomini & $3.4 \times 10^{5}$ & $0.0625,-0.3517,0.7363,-0.5658,0.0975,0.0244$ \\
\hline Prinsen & - & $0.0234,-0.2065,0.6238,-0.6940,0.2909,-0.0377$ \\
\hline Binom & - & $0.0630,-0.3150,0.6299,-0.6299,0.3150,-0.0630$ \\
\hline
\end{tabular}

TABLE III

PERFormancE COMPARISON (INTERPUlse PERIODS ARE FROM [2])

\begin{tabular}{|l|c|c|c|}
\hline & Stop. Att. $(\mathrm{dB})$ & Mean Pass. Err. (dB) & Max. Dev. $(\mathrm{dB})$ \\
\hline LS & -30.66 & -0.6012 & 22.32 \\
\hline Min-Max & $\mathbf{- 3 6 . 5 5}$ & $\mathbf{- 0 . 5 6 9 6}$ & $\mathbf{1 7 . 9 5}$ \\
\hline Hsiao & -31.33 & -0.5704 & 21.37 \\
\hline Prinsen & -15.6 & -0.4922 & 16.87 \\
\hline Binom & -14.57 & -0.5133 & 20.23 \\
\hline
\end{tabular}

TABLE IV

PERFORMANCE COMPARISON (INTERPULSE PERIODS ARE FROM [3])

\begin{tabular}{|l|c|c|c|}
\hline & Stop. Att. (dB) & Mean Pass. Err. (dB) & Max. Dev. (dB) \\
\hline LS & -39.61 & -0.02622 & 10.41 \\
\hline Min-Max & $\mathbf{- 4 7 . 9 1}$ & $\mathbf{- 0 . 2 5 1 2}$ & $\mathbf{1 2 . 6 4}$ \\
\hline Jacomini & -38.25 & -0.01394 & 10.88 \\
\hline Prinsen & -40.55 & -0.2161 & 38.66 \\
\hline Binom & -24.0 & -0.2436 & 53.72 \\
\hline
\end{tabular}

Figures 6 and 7 present the comparison of the improvement factor performance of the filters with the optimum filter whose coefficient vector is calculated as in (18) for each particular Doppler frequency of interested frequency interval.

$$
\mathbf{w}_{\text {opt }}=\mathbf{R}^{-\mathbf{1}} \mathbf{s}
$$

Here $\mathbf{w}_{\mathbf{o p t}}$ is the coefficient vector of optimum filter, $\mathbf{R}^{-\mathbf{1}}$ inverse of the clutter covariance matrix and $\mathbf{s}$ is the signal vector which is

$$
\left[\begin{array}{lllll}
1 & e^{j 2 \pi f t_{1}} & e^{j 2 \pi f t_{2}} & \ldots & e^{j 2 \pi f t_{N-1}}
\end{array}\right]
$$

where $t_{k}$ is defined as in (2).

Uniform clutter power spectrum density (PSD) is used in these comparisons as in [2], [3]. If Figure 6 is examined, the first important point is the difference between Prinsen's and Binomial filters in comparison to others. There is nearly 20 $\mathrm{dB}$ difference in average as compared to the optimum filter's improvement factor. The reason for this difference is the poor attenuation performance of these filters for wider stopband. Performance of the other filters close to each other. The best value of improvement factor is attained with Hsiao's filter.

The improvement factor performance of the filters in Figure 7 is similar to each other except the Binomial filter. Due to uniform clutter PSD and wider stopband, it exhibits poor performance. Minmax filter provides the best improvement factor performance compared to the others. Flat response of the minmax design in the passband can be thought as the reason for better performance.
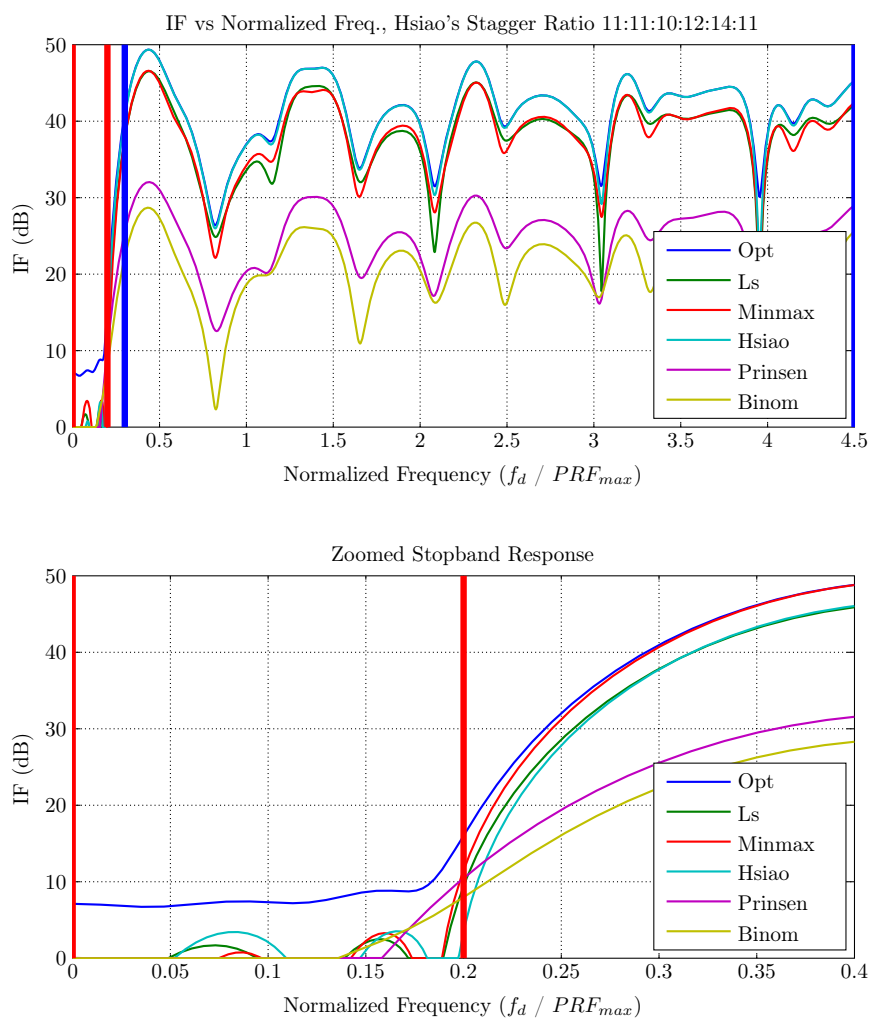

Fig. 6. Improvement Factor Comparison of Filter Designs with [2].
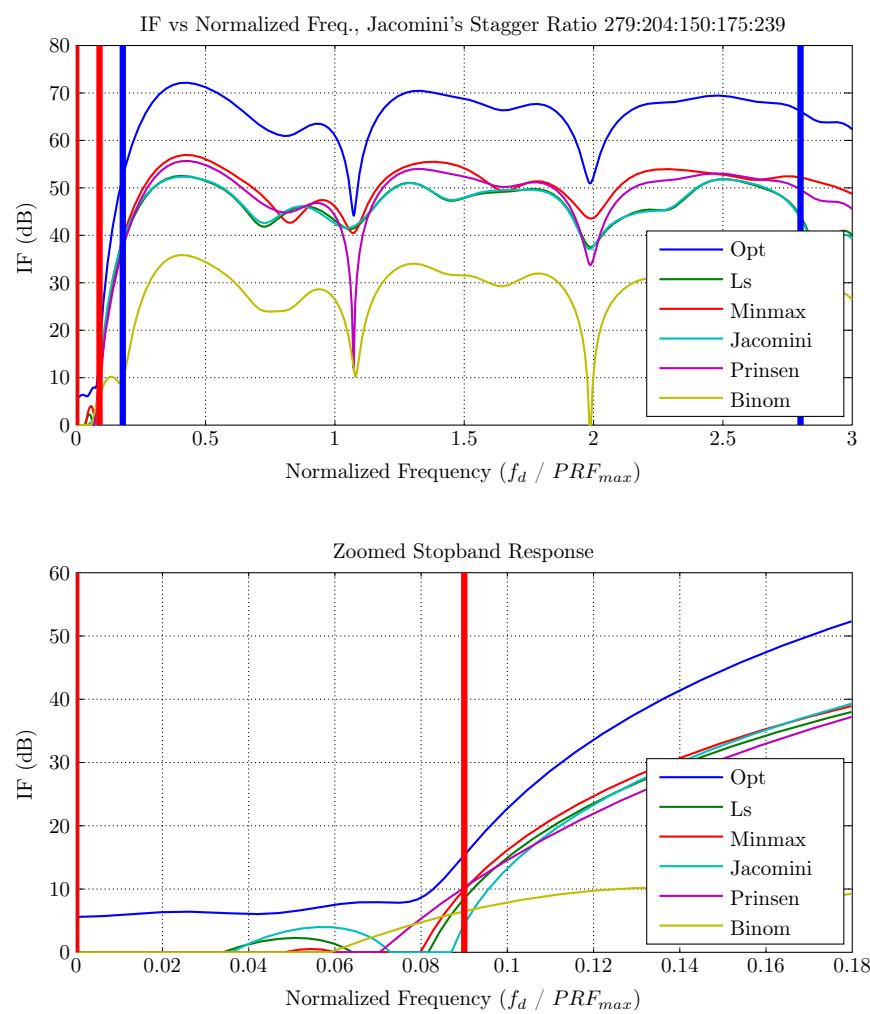

Fig. 7. Improvement Factor Comparison of Filter Designs with [3]. 
TABLE V

IF VALUES FOR COMPARISON OF DESIGNED FILTERS WITH [2]

\begin{tabular}{|c||c|c|c|c|c|c|}
\hline & Optimum & Ls & Min-Max & Hsiao & Prinsen & Binom \\
\hline \hline IF (dB) & 39.606 & 36.222 & 36.172 & 38.900 & 21.749 & 19.667 \\
\hline
\end{tabular}

TABLE VI

IF VALUES FOR COMPARISON OF DESIGNED FILTERS WITH [3]

\begin{tabular}{|c||c|c|c|c|c|c|}
\hline & Optimum & Ls & Min-Max & Jacomini & Prinsen & Binom \\
\hline \hline IF (dB) & 63.123 & 44.575 & 48.447 & 44.660 & 46.069 & 27.209 \\
\hline
\end{tabular}

Another notable result is the similarity between the performances of the Jacomini's and the least square filter. This result is expected, since the cost functions of the designs built onto same objectives. Despite the difference between the underlying analytical formulations, similar scenario parameters and frequency boundaries lead to similar improvement factor results.

\section{CONCLusion}

This paper examines the problem of MTI filter design for staggered MTI systems. The weighted least square and minmax filter design techniques are utilized to design filters with nonuniform sampling. It has been shown that with a proper selection of a weight parameter, a good compromise between clutter attenuation and flat passband response can be attained. The results of this paper can be especially important for weather surveillance systems where staggered MTI is frequently utilized. For many practical applications LS design can provide reasonable performance. On the other hand, minmax design should be carefully studied, since it has many local minimas. If flatter passband is desired and sufficient computer power is available, minmax design can be more suitable.

It should be noted that, the usage of optimal MTI filter can provide better clutter attenuation and passband performance compared to other designs. However, optimal filter specific for each Doppler frequency of interest must be calculated.

\section{REFERENCES}

[1] P. Prinsen, "A class of high-pass digital MTI filters with nonuniform PRF," Proc. IEEE, vol. 61, no. 8, pp. 1147-1148, Aug. 1973.

[2] J. Hsiao and F. Kretschmer Jr, "Design of a Staggered-PRF MTI Filter," DTIC Document, Tech. Rep., 1973.

[3] O. Jacomini, "Weighting Factor and Transmission Time Optimization in Video MTI Radar," IEEE Trans. Aerosp. Electron. Syst., vol. AES-8, no. 4, pp. 517-527, July 1972.

[4] P. Prinsen, "Elimination of Blind Velocities of MTI Radar by Modulating the Interpulse Period," IEEE Trans. Aerosp. Electron. Syst., vol. AES-9, no. 5, pp. 714-724, Sept. 1973.

[5] Z. Banjanin and D. Zrnic, "Clutter rejection for Doppler weather radars which use staggered pulses," IEEE Trans. Geosci. Remote Sens., vol. 29, no. 4, pp. 610-620, July 1991.

[6] B. Bidégaray-Fesquet, L. Fesquet et al., "A fully nonuniform approach to FIR filtering," in SAMPTA'09, International Conference on Sampling Theory and Applications, Marseille, France, Feb. 2009.

[7] Z. Jin, X.-D. Li, and J. King, "Non-uniform time sampling FIR filter design based on least-squares criterion," Electronics Letters, vol. 46, no. 23, pp. 1544-1545, 112010.
[8] C. Nguyen, D. Moisseev, and V. Chandrasekar, "A time domain clutter filter for staggered PRT and dual-PRF measurements," in Geoscience and Remote Sensing Symposium, 2007. IGARSS 2007. IEEE International, July 2007, pp. 3325-3328.

[9] W. Zuyin, "Optimal design of clutter rejection filters for MTI system," in Radar, 2001 CIE International Conference on, Proceedings, 2001, pp. $475-478$.

[10] G. Ewell and A. Bush, "Constrained Improvement MTI Radar Processors," IEEE Trans. Aerosp. Electron. Syst., vol. AES-11, no. 5, pp. 768 780, Sept. 1975.

[11] S. Gilon and A. Venetsanopoulos, "Design of MTI radar on the basis of detection probability," Aerospace and Electronic Systems, IEEE Transactions on, vol. AES-15, no. 1, pp. 106 -118, Jan. 1979.

[12] "IEEE standard radar definitions," IEEE Std 686-2008 (Revision of IEEE Std 686-1997), pp. c1 -41, 212008.

[13] M. İspir, "Design of moving target indication filters with non-uniform pulse repetition intervals," Master's thesis, Middle East Technical University (METU), Department of Electrical-Electronics Engineering, Jan. 2013.

[14] S.-P. Wu, S. Boyd, and L. Vandenberghe, Applied Computational Control Signal and Communications. Birkhauser, 1997, ch. 1: FIR Filter Design via Spectral Factorization and Convex Optimization, pp. 1-33. 